\title{
Ausblick auf Revolutionen des Lesens
}

Roger Chartier und Guglielmo Cavallo haben in ihrer Welt des Lesens ${ }^{1}$ betont, dass sich eine Geschichte des Lesens nicht auf jenen Zeitraum beschränken dürfe, als allein mit den Augen und leise zu lesen begonnen wurde. Denn dies sei nur eine unter vielen Modalitäten des Lesens, deren Geschichte sich leicht in ihrer historischen Entwicklung nachzeichnen lasse. Es kann im Rahmen unserer Vorlesung freilich nicht darum gehen, eine wirkliche und wahrlich umfangreiche Geschichte des Lesens nachzuvollziehen, die von der Antike bis in die Gegenwart reichen würde und den Weg von den Schriftrollen, den volumina, dem Papyros, über den Codex mit Buchseiten - Julius Cäsar soll der erste gewesen sein, der Schriftrollen in einzelne Seiten für seine Truppen binden ließ - und viel später die Gutenberg'sche Druckerpresse des 15. Jahrhunderts, bis hin in die Gegenwart des Taschenbuches und der Papierseiten sowie der Computerbildschirme und deren scrollende Schriftrollen zu verfolgen. Eine solche Geschichte ist faszinierend, aber ihre Historie ist sehr gut aufgearbeitet und hätte mit unserem Fach, der Romanistik und Komparatistik, wenig zu tun. Konzentrieren wir uns also auf einige jener Elemente, die für unseren Themenkreis von Belang sind.

In den Ländern des Abendlandes entwickeln sich zwischen dem 16. und dem 19. Jahrhundert die Lesepraktiken durchaus unterschiedlich, wie Chartier und Cavallo betonten: je nach dem Stand der industriellen und gesellschaftlichen Entwicklung, der Alphabetisierung und vieler weiterer Parameter, die den Entwicklungsstand der westlichen Gesellschaften beschreiben.

Die Akten der Inquisition in den Ländern des Mittelmeeres - und insbesondere in Spanien - geben uns einen hervorragenden Aufschluss über bestimmte Lesepraktiken, aber auch Formen des Zugangs zum Buch und weiteren Darbietungsformen von Wissen. Denn dieser Zugang war strikt festgelegt und geregelt vergessen wir nicht, dass etwa Frauen, um nur ein Beispiel herauszugreifen, die Benutzung der lateinischen Sprache und damit der Zugang zu den damaligen Wissensbeständen der Wissenschaft streng verboten war. Nicht allein im iberischen Mutterland, sondern gerade auch in den Kolonialgebieten wurden vielfältige Erklärungen von Angeklagten zu den von ihnen gelesenen und von der Inquisition inkriminierten Werken gesammelt. Die Akribie der Inquisitoren, möglichst viele Details über diese Praktiken zu sammeln, bietet eine nahezu perfekte Grundlage für deren wissenschaftliche Auswertung.

1 Vgl. Chartier, Roger / Cavallo, Guglielmo (Hg.): Die Welt des Lesens. Von der Schriftrolle zum Bildschirm. Frankfurt am Main - New York - Paris: Campus Verlag - Editions de la Maison des Sciences de l'Homme 1999, S. 12.

Ә Open Access. (C) 2020 Ottmar Ette, publiziert von De Gruyter. @B)BY-NC-ND Dieses Werk ist lizenziert unter der Creative Commons Attribution-NonCommercial-NoDerivatives 4.0 International Lizenz. 

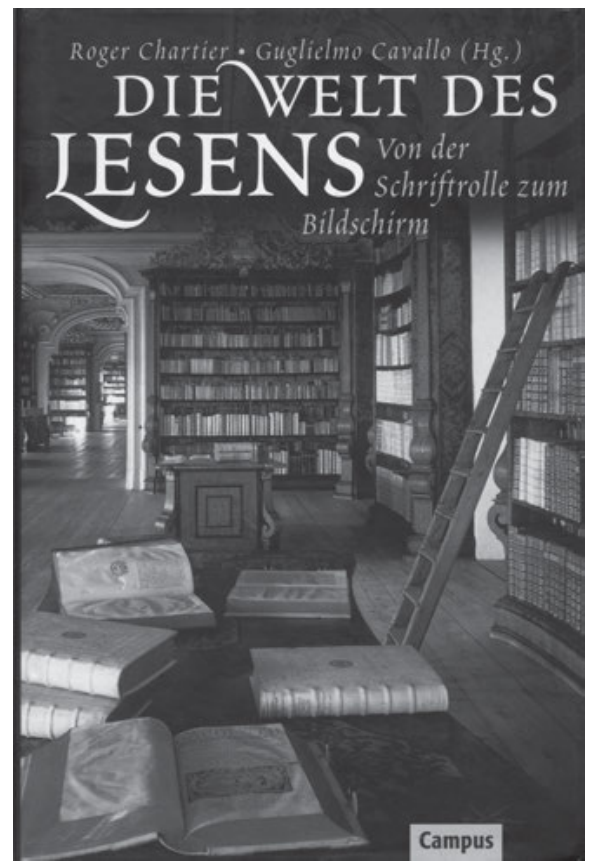

Abb. 30: Roger Chartier (Hg.), Guglielmo Cavallo (Hg.): Die Welt des Lesens. Von der Schriftrolle zum Bildschirm, Cover.

Im europäischen Puritanismus sind es wiederum die spirituellen Autobiographien und die pietistischen Lebensbeichten, die aufschlussreiche Zeugnisse über die Lesegewohnheiten ihrer Verfasser liefern können. Im Verlauf des 18. Jahrhunderts entstehen dann auf Grund der industriellen Entwicklung neue Märkte für das gedruckte Buch, neue Leserschichten und letztlich auch neue Lesepraktiken, die wir uns etwas näher anschauen wollen.

Man kann mit Roger Chartier und Guglielmo Cavallo von drei großen Revolutionen des Lesens in der Neuzeit sprechen. ${ }^{2}$ Die erste der profunden Transformationen, welche die Lesepraktiken betreffen, ist dabei technischer Natur. Denn in der Mitte des 15. Jahrhunderts erfolgte eine Revolutionierung durch das Prinzip der beweglichen Lettern und der Druckerpresse, so dass seither die handschriftliche Abschrift nicht mehr die einzige Möglichkeit war, die Vervielfältigung und Zirkulation von Texten zu gewährleisten. Mit dieser bahnbrechenden Erfindung ging eine Senkung der Kosten und zugleich eine deutliche Verminderung der Herstellungsdauer gedruckter Texte einher, außerdem die Tatsache, dass die so gedruckten Bücher miteinander identisch, textgleich waren und keine individuellen Schreibfehler und Lesefehler mehr vorkamen. Einmal abgesehen von

2 Ebda., S. $39 \mathrm{ff}$. 
manchen Ficciones von Jorge Luis Borges. Die Gutenberg-Galaxis war entstanden, und mit ihr eine der technischen Grundlagen der rasanten Entwicklung des Abendlandes.

Jedoch hob das französisch-italienische Forscherteam auch hervor, dass allein dies noch keine Revolution der Lesepraktiken mit sich gebracht hätte. Es lässt sich allerdings trefflich darüber streiten, ob die neuen Techniken in der Gutenberg-Galaxis an den Grundstrukturen des Buches - wie die beiden Autoren behaupteten - wirklich nichts zu ändern vermochten. Denn immerhin - und dies lässt sich in einer Vielzahl von Texten nachweisen - wurde durch die Druckerpresse die Handschrift und damit die Körperlichkeit des Schreibenden ausgeschlossen und zugleich entindividualisiert. Denn die metallenen Lettern waren nun für alle gleich - unabhängig davon, ob sie ihre Texte diktierten, ob sie sie auf Papier niederschrieben und welche Handschrift sie dabei verwendeten: Ihr Körper war aus den Bewegungen der Schrift fortan ausgeschlossen.

Dies ist auch bei den weiteren technischen Implementierungen auf Ebene der Niederschrift von Manuskripten der Fall: Während in der Handschrift noch der gesamte Körper-Leib sichtbar und anschaulich ist, können wir ihn in der Schreibmaschine doch noch immerhin von Spezialisten in bestimmten Anschlagweisen rekonstruieren. Es gab früher noch Kriminologen, welche die Schreibmaschinenschrift zu analysieren wussten. Doch in Ihren heutigen Computern oder Smart Phones ist kein Unterschied mehr sichtbar, egal, ob sie das „e“ der Tastatur mit besonderer Vehemenz drücken oder nicht: Das Ergebnis ist immer gleich, Ihr Körper ist ausgeschlossen.

Diesem Ausschluss des Körpers aus Seiten der Schreibenden entspricht auch ein Ausschluss des Körpers auf Seiten der Lesenden zumindest insoweit, als der scriptor nun nicht mehr mit seinem eigenen Körper präsent ist. Zugleich verbleiben durch die gedruckte Schrift nur noch ganz bestimmte und kleine Stellen, an denen der lector, oder auch commentator, eigene persönliche Reflexionen an den Rand schreiben und damit zu Protokoll geben kann. Wir wissen, dass sich dies bei elektronischen Texten erneut gewandelt hat und wir heute nicht nur über umfangreiche Korrekturfunktionen verfügen, sondern gleichsam halbe Romane in das Geschriebene vormaliger Verfasser einfügen können. Gewiss haben Chartier und Cavallo recht mit ihrer Behauptung, dass das Buch vor und nach Gutenberg durchaus recht ähnlich strukturiert war. Denn die Untergliederungen und Einteilungen der Bücher sind kein Ergebnis des Buchdrucks, sondern zu einem viel früheren Zeitpunkt eingeführt worden. Schon zu Zeiten der Handschrift verbreiten sich Inhaltsverzeichnisse, Indices, Glossare usw.; und selbst eine genaue Rangfolge der Formate setzte sich in den letzten Jahrhunderten der Handschrift - und nicht erst nach der Einführung des Buchdrucks - durch: vom Groß-Folio-Format abwärts. Der Buchdruck trug 
all diese Innovationen in sein eigenes Universum ein und hob diese Errungenschaften damit zugleich in ein neues Zeitalter. So können wir heute, um nur ein Beispiel zu nennen, nach bestimmten Elementen eines Inhaltsverzeichnisses unsere Texte durchsuchen oder auch verändern.

Die erste eigentliche Leserevolution der Neuzeit ist also weitgehend unabhängig von der technischen Revolution, die im 15. Jahrhundert die Buchherstellung so radikal verändert hatte. An dieser historischen Schnittstelle gilt es, die enorme Wichtigkeit des Übergangs vom monastischen zum scholastischen Lesemodell zu berücksichtigen, denn hier erst wird das Buch zum Gegenstand und Werkzeug geistiger Arbeit. Denn anders als beim monastischem Lesen, bei dem es darum ging, wenige Bücher möglichst intensiv zu kennen und immer wieder von neuem zu lesen, ja auch auswendig zu lernen, geht es beim scholastischen Lesen um eine möglichst breite Kenntnis unterschiedlichster Schriften, wobei die Bücher dabei die eigentlichen Arbeitsinstrumente - und nicht statische Quellen der Erkenntnis - sind. Dies ist eine in ihren Konsequenzen fundamentale Veränderung, die noch für uns heute von erheblicher Bedeutung ist.

So war das gleichzeitige Lesen verschiedener Bücher bereits in der Scholastik als Lesepraxis aufgekommen. Ich weiß nicht, ob Sie immer erst einmal in einem Buch lesen und es abschließen, bevor Sie zu einem anderen übergehen, oder ob Sie zu den Leserinnen und Lesern zählen, die zwei oder mehrere Bücher gleichzeitig lesen und von einem ins andere wechseln. Fakt ist, dass Sie bei der Praxis eines Lesens in mehreren Büchern durchaus schon auf eine lange Lesegeschichte zurückblicken können. Denn früh schon wurden auch sogenannte Bücherräder oder Leseräder verwendet, die ein bequemes gleichzeitiges Lesen unterschiedlicher Bücher ermöglichten beziehungsweise erleichterten.

Im Humanismus verstärkte sich diese Tendenz und Lesepraxis noch; und so habe ich Ihnen auch die Abbildung der von Ramelli erfundenen raffinierten Lesemaschine aus dem Jahre 1588 mitgebracht. ${ }^{3}$ Sie steht im Grunde nur für eine veränderte Lesepraxis, die sich weit vom monastischen Modell entfernt hat - und in gewisser Weise sind etwa philologische Lesepraktiken hier historisch auch verankert. Verstehen Sie mich bitte nicht falsch: Damit ist keinerlei Wertung verbunden. Und die Ein-Buch-Lektüre vermag mindestens genauso intensiv zu sein wie die Art und Weise, mehrere Bände gleichzeitig zu verschlingen.

3 Vgl. Manguel, Alberto: Eine Geschichte des Lesens, S. 157. 


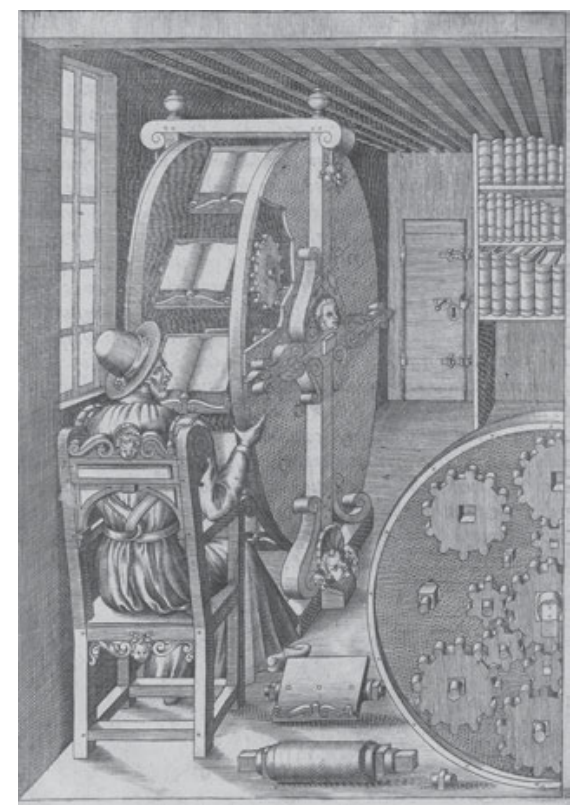

Abb. 31: Ramellis Bücherrad aus dem 16. Jahrhundert.

Damit ging eine Entwicklung hin zum stillen Lesen einher, mit der wir uns ja bereits beschäftigt haben. Mit diesem stillen Lesen ist eine grundlegende Zäsur verknüpft, welche auch das parallele Lesen in großen Gelehrtengemeinschaften ermöglicht. Denn was wäre ohne die Kulturpraxis des stillen Lesens ein Lesesaal, sagen wir in der Staatsbibliothek? Das stille Lesen stellt eine Beziehung zum Geschriebenen her, die freier, auch geheimer, und in jedem Falle völlig innerlich sein kann. ${ }^{4}$ Dies war in der Tat eine wirkliche, aber stille Revolution des Lesens und der Lektüre.

Die zweite Leserevolution der Neuzeit verdankt sich ganz offensichtlich der industriellen Buchproduktion. In der zweiten Hälfte des 18. Jahrhunderts - so behauptet man - soll auf das intensive Lesen ein anderes Lesen gefolgt sein, das im Allgemeinen als extensiv bezeichnet wird. Der intensive Leser verfügte über ein genau definiertes, von Generation zu Generation weitergereichtes Korpus von Büchern, die gelesen und wiedergelesen wurden. Der Kanon stand nicht in Frage, sondern war die Norm, an der man sich orientierte.

Dies ist, um es mit der heutigen Lage und den aktuellen Bedingungen des Studierens zu vergleichen, eine gänzlich andere Situation, haben die neuen Studierendengenerationen doch keinen Bezug mehr zu einer Liste von Werken, die

4 Ebda., S. 47. 
unbedingt im Verlauf des Studiums gelesen werden müssen. In den neunziger Jahren noch habe ich am Institut für Romanistik eine Leseliste eingeführt und ging dabei von der Vorstellung aus, dass man als Studierende an jedem Wochenende im Jahr ein Buch zu Ende lesen könne. So hatte ich nach Anweisung eines meiner Dozenten mein eigenes Studium auch aufgebaut. Wie dem auch sei: Auf diese Weise wären innerhalb kürzester Zeit die hundert oder hunderfünfzig Werke der Leseliste tatsächlich zu lesen gewesen. Doch die heutigen Zeiten sind derartigen Praktiken nicht mehr günstig gesonnen. Denn der Bologna-Prozess, der heute selbst von jenen kritisiert wird, die ihn eingeführt haben - ein im Übrigen eigenartiger Tatbestand von Fahrerflucht, der freilich nicht geahndet wird -, sowie die Vielzahl an unterschiedlichen Studiengängen, die studiert werden können, erschweren - verbunden mit den auf das gesamte Studium verteilten Prüfungsleistungen - und der dauernden Anspannung aufgrund eines beständigen workloads jegliche Orientierung an einem Lesekanon. Dies gilt es nicht zu beklagen, sondern zu konstatieren. Das heutige Studium und die damit verbundenen Lesepraktiken haben sich zumindest für die Mehrzahl der Studierenden erheblich gewandelt. Und die Medien, in denen gelesen wird, selbstverständlich auch.

Aber kommen wir zurück auf die Unterscheidung zwischen einem intensiven und einem extensiven Lesertypus. Denn anders als bei der intensiven Lektüre waren die extensive Leserin, der extensive Leser von einer Lesewut getrieben, wie sie sich in der Goethe-Zeit Deutschlands bemächtigt zu haben scheint. Man spricht von einem freien, unehrerbietigen, respektlosen Lesen, das ständig die Druckerzeugnisse wechselte und hemmungslos konsumierte. Wir werden dafür noch ein literarisches Beispiel kennenlernen, das uns genau in diese Zeit führt und zugleich mit der Liebesthematik verbandelt ist.

Doch lassen sich diese klassischen Thesen im Lichte neuerer Forschungen und Untersuchungen wohl nicht mehr länger aufrechterhalten. Denn durchaus zahlreich sind die extensiven Leser zur Zeit der intensiven Lektüre - wofür einmal mehr das Bücherrad, aber auch die Sammlungen von Gemeinplätzen stehen mögen. Genau zeitgleich mit dieser Leserevolution entfaltet sich mit Rousseau, Bernardin de Saint-Pierre oder Richardson eine höchst intensive Lektüre, durch die sich der Roman des Lesers bemächtigt und ihn in seinen Bann und in seine Abhängigkeit zwingt. Wir werden auch dafür noch mehrfach Beispiele finden, doch erscheint es mir an dieser Stelle schon sinnvoll, erneut auf einen Roman Italo Calvinos zu verweisen, in dem diese Lesewut, die ins Unheil führen kann, deutlich wird: Il barone rampante, der Baron auf den Bäumen, mit dem wir uns noch näher beschäftigen werden. Und ich bitte Sie schon an dieser Stelle um Vergebung dafür, dass ich noch ein zweites Mal Italo Calvino bemühen werde, ist sein Roman doch mehr als jeder andere dazu geeignet, uns für diese Leserevolution - aber auch für die Liebeskonzeptionen der Zeit - die Augen zu öffnen. 
Große Romane dieser Zeit des 18. Jahrhunderts wie Pamela, Julie ou la Nouvelle Héloïse, Paul et Virginie oder der Werther werden ständig gelesen, wiedergelesen, zitiert und rezitiert, wobei eine Identifikation des Lesepublikums mit den Figuren des jeweiligen Romans erfolgt und das eigene Leben im Lichte der literarischen Gestalten gedeutet wird. Bei dieser identifikatorischen Lesart, bei dieser intensiven Lektüre neuen Typs wird nun die ganze Gefühlswelt in Anspruch genommen: Tränen und Gefühlsausbrüche können kaum zurückgehalten werden, die Lektüre ist notwendig mit starken Emotionen verbunden. Dabei schreibt man auch häufig an den Verfasser, der mit seinen Werken zu einem wahren Lebensberater geworden ist und durchaus in Kontakt mit seinem zeitgenössischen Lesepublikum steht. Auch an dieser längst historisch gewordenen Lesesituation zeigt sich also, dass das Buch bei einer derart intensiven Lektüre unmittelbar ein Lebenswissen zu enthalten scheint, das sich eine zunehmend breiter gefächerte Leserschaft aneignen und einverleiben will. Wir stoßen bei diesen Lesarten unzweifelhaft auf jene Dimension, mit der wir uns immer wieder beschäftigen werden: Literatur als Lebenswissen.

Halten wir also fest: Belege für eine wirkliche Leserevolution in der zweiten Hälfte des 18. Jahrhunderts lassen sich zumindest in England, Frankreich und Deutschland leicht finden. Die entscheidenden Faktoren dafür sind eine wachsende Buchproduktion, die sich von Beginn des 18. Jahrhunderts bis in die 1780er Jahre verdreifacht bis vervierfacht, die rasche Zunahme der Tageszeitungen, ein deutliches Sinken des Buchpreises, die Zunahme von Lesegesellschaften, Leihbibliotheken und Clubs. Das Lesen am Ausgang des 18. Jahrhunderts ist nicht mehr das, was es zu Beginn dieses Jahrhunderts gewesen war. So bildet das Jahrhundert der industriellen (ausgehend von England) sowie der politischen Revolution (ausgehend von Frankreich) auch das Jahrhundert einer Leserevolution, welche in mehr als einem Sinne die Moderne im letzten Drittel des 18. Jahrhunderts eröffnet.

Die dritte Revolution des Lesens, Sie haben es schon kommen sehen, fällt nun in unsere Tage. Sie betrifft die elektronische Speicherung und Übertragung der Texte und die durch sie diktierte Art des Lesens, die sich grundlegend verändert hat. Denn auf einem Bildschirm, auf einem screen (sei es der Ihres Computers oder der Ihres Smart Phones) zu lesen ist anders, als in einem Codex, auf Papyrus oder auch auf Papier in einem Buch zu lesen.

Dabei verändert sich nicht zuletzt der Kontextbegriff, insoweit Texte nun innerhalb logischer Architekturen positioniert werden, etwa innerhalb elektronischer Datenverwaltungen oder Datenbanken, innerhalb von zur Verfügung gestellten oder autonomen Sucharchitekturen oder auch von hyper- und metatextuellen Verweissystemen. So gerät das ganze bisherige System der Erkennung und Handhabung, der Zettelkästen und Recherchen von Daten aus den Fugen oder genauer: Es rückt in neue Fugen ein. Diese Revolution vollzog sich innerhalb 
von weniger als einer Generation. Sie würden lachen, wenn ich Ihnen erzählte, dass ich noch mit der Hand schrieb und eine Reiseschreibmaschine bediente, als ich mein Studium aufnahm, und dass ich als junger Student (so nannte man das damals) eine Einführung in die Bibliographie und das bibliographische Arbeiten erhielt von einem Mann, der eigens für derartige Einführungen eingestellt war und an dem der Staub von Hunderten von Zettelkästen buchstäblich klebte.

In gewisser Weise - und durchaus paradoxerweise - findet man einiges von der aktuellen Stellung des Lesers in der Antike wieder, der ein volumen, eine Schriftrolle las, die er sukzessive ent-wickelte oder eben scrollte. Gleichzeitig aber ist der Text auf Ihrem Computerbildschirm, auf Ihrem iPad oder Ihrem Smart Phone mit einer Seitennumerierung und mit allen Markierungen eines Codex ausgestattet, so dass man hier mit Chartier und Cavallo sehr wohl von der „Kreuzung zweier Logiken" sprechen kann. ${ }^{5}$

Zugleich wird die Gleichzeitigkeit von Produktion, Übermittlung und Lektüre ein- und desselben Textes möglich: Die getrennten Aufgaben des Schreibens, Herausgebens und Verteilens sind nicht länger voneinander getrennt. Unterschied man in der Antike oder im Mittelalter der Scholastik noch zwischen scriptor, compilator, commentator und auctor, so haben sich in der aktuellen Welt diese Funktionen von Textinstanzen nicht nur deutlich verändert: sie haben sich jenseits alter Grenzziehungen viel stärker miteinander vermischt und vermengt. Als Herausgeber einer Hybrid-Edition historischer Schriften an der BerlinBrandenburgischen Akademie der Wissenschaften kann ich Ihnen verraten, dass die verschiedenen Autor- oder Herausgeberfunktionen, aber auch die Textfunktionen selbst in einem erheblichen Maße schwanken, je nachdem, ob man sich im Printbereich oder im Digitalbereich befindet. Dass wir für unsere Hybrid-Edition das Digitale als Grundlage gewählt haben, ist heute noch innovativ, entspricht aber durchaus den sich im Aufbau befindlichen Standards der Digital Humanities. Diese Standards aber werden sich ihrerseits wieder verändert haben, bis in etwa fünfzehn Jahren unser Projekt an ein vorläufiges Ende gelangt.

All dies hat ebenso Folgen für rechtliche Kategorien wie das sich in einer öffentlichen Debatte befindliche Copyright, für philologische Kategorien wie die Vollständigkeit, Beständigkeit eines Textes, oder für ästhetische Kategorien wie die Originalität, die ikonotextuelle oder Faksimile-Darstellung, aber auch für normative Kategorien wie die Ablieferung von Pflichtexemplaren usw. Es wäre müßig, an dieser Stelle die Vor- und Nachteile von Open Access nicht nur für die „Bücher“, sondern auch für die Verlagslandschaft selbst zu diskutieren, in welcher diese Bücher entstehen. Waren die bibliophilen Nachlässe von

5 Chartier, Roger / Cavallo, Guglielmo (Hg.): Die Welt des Lesens, S. 45. 
Gelehrtenbibliotheken einstmals Gegenstand hitziger Begehrlichkeiten und entbrannten um sie wahre Kämpfe, so winken heutige Bibliotheken müde ab, wenn wieder einmal eine Privatbibliothek verkauft werden soll: Die Kosten für die Erhaltung papierener Buchbestände rechnet sich in Zeiten digitaler Archivierung längst nicht mehr. Allerdings unabhängig von der Tatsache, dass noch immer die Lesbarkeit dieser Bestände für eine längere Zukunft nicht garantiert ist. Denn unter diesen Voraussetzungen sind die Tontafeln des Gilgamesch-Epos nach wie vor das herausragende Medium, das selbst noch nach Jahrtausenden - und nach vielen mächtigen Leserevolutionen - schlicht akzessibel und lesbar geblieben ist.

Kuba-Tagebuch: Edition forschungscossier

\section{Isle de Cube. Antilles en général}

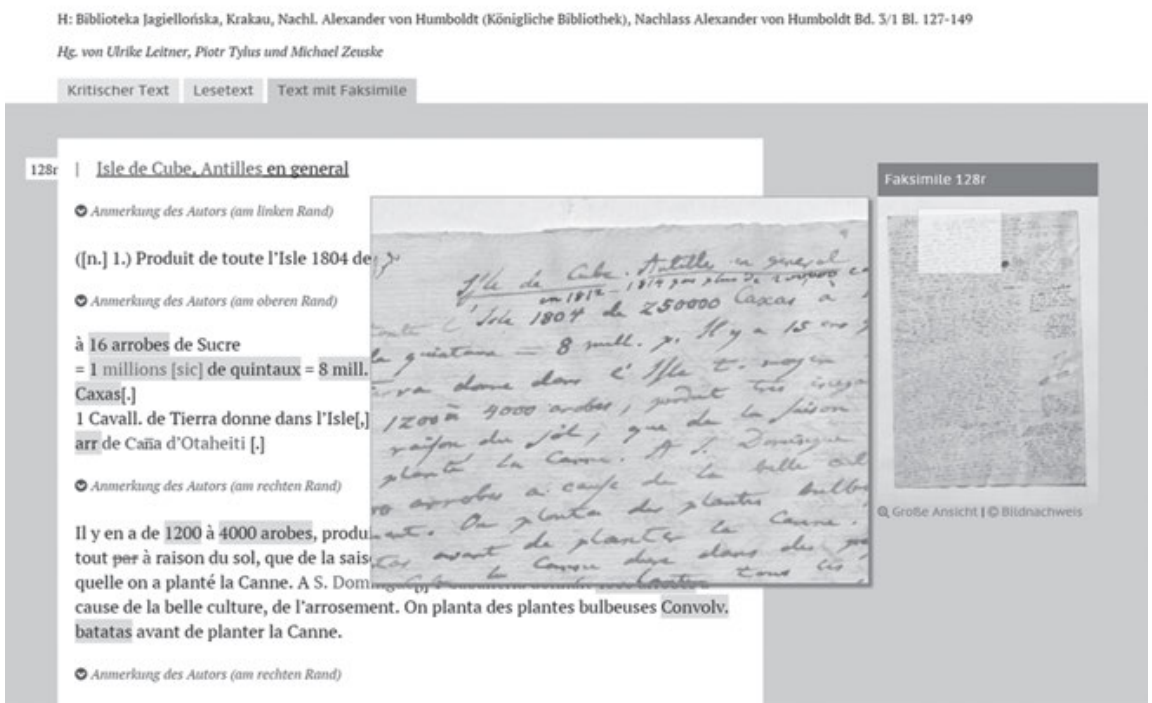

Abb. 32: Digitale Kartierung eines Tagebuchs, Bildschirmfoto aus der Berliner „edition humboldt digital“ der Berlin-Brandenburgischen Akademie der Wissenschaften.

Damit verschwinden zugleich auch zwei grundlegende Bedingungen, die zuvor galten: Erstens waren die Eingriffe der Leserschaft in das Buch beziehungsweise den Text streng beschränkt geblieben; sie kann im digitalen Zeitalter aber gleichsam zum Ko-Autor des Textes avancieren. Überhaupt ist die Funktion des literarischen Autors mehr als brüchig geworden. Und zweitens wird nun der Widerspruch aufgehoben, der schon den Menschen der Antike plagte: der Widerspruch zwischen dem Traum von einer universalen Bibliothek und der 
zwangsläufig enttäuschenden Wirklichkeit tatsächlich und real existierender Bibliotheken - und ich sage dies nicht nur mit Blick auf unsere Universitätsbibliothek in Potsdam, die sich im Verlauf der zurückliegenden Jahre freilich verbessert hat. Internetgestützt kann ich mir riesige Universalbibliotheken auf mein Smart Phone holen - und die damit verbundene Demokratisierung des Wissens ist ein herausragender, wenn auch freilich nur ein Aspekt unter vielen. Die elektronische Vernetzung hebt die bislang zwangsläufige Verbindung zwischen dem Ort des Textes und dem Ort von Leserin und Leser auf, so dass der alte Traum von der universalen Bibliothek - einen Zugang zum Netz vorausgesetzt - sich zunehmend verwirklicht oder auch schon verwirklicht hat.

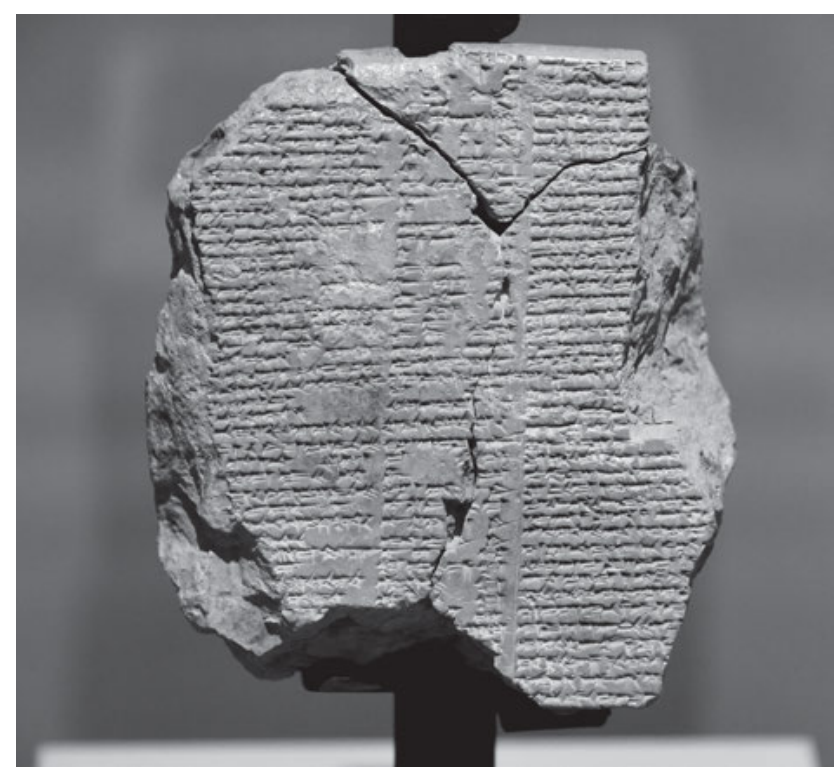

Abb. 33: Tontafel V des Gilgamesch-Epos.

Soviel also zu den drei Revolutionen, die das Lesen im Abendland seit dem Mittelalter geprägt und strukturiert haben. Wir leben heute in einer Zeit des Umbruchs, gerade auch, was Lektürepraktiken und damit ein fundamentales kulturelles Praxiswissen angeht. Ich möchte Ihnen dies abschließend vermittels eines Zitats von Cavallo und Chartier vorführen:

Wie Armando Petrucci zeigt, ist die traditionelle Lektüre in unserer heutigen Welt der Konkurrenz durch das Bild ausgesetzt und zugleich der Bedrohung, ihre durch schulische und gesellschaftliche Normen vermittelten Repertoires, Regeln und Verhaltensweisen zu 
verlieren. $\mathrm{Zu}$ diesem Umbruch kommt ein zweiter, der vorerst noch kaum ausgeprägt ist und je nach Land unterschiedlich wahrgenommen wird: dass nämlich der Träger des Geschriebenen verändert und der Leser plötzlich zu neuen Gesten und neuen intellektuellen Praktiken gezwungen ist. Der Schritt vom Kodex zum Bildschirm ist ebenso bedeutend wie der von der Schriftrolle zum Kodex. Mit ihm wird die Ordnung der Bücher, die den Männern und Frauen des Abendlandes seit den ersten Jahrhunderten des christlichen Zeitalters vertraut war, in Frage gestellt. So setzten sich neue Arten des Lesens durch oder drängen sich auf, die man in ihrer Gesamtheit noch nicht kennzeichnen kann, die jedoch zweifellos grundlegend neue Lesepraktiken mit sich bringen. ${ }^{6}$

Doch tauchen wir jetzt wie versprochen ein in die neuen Lese- und Liebespraktiken, die sich im Verlauf der zweiten Hälfte des 18. Jahrhunderts herauskristallisierten. Dazu wollen wir erneut auf ein Beispiel aus der italienischen Literatur zurückgreifen.

6 Chartier, Roger / Cavallo, Guglielmo (Hg.): Die Welt des Lesens, S. 47f. 